\title{
Anchoring properties of photoaligned azo-dye materials
}

\author{
Vladimir Chigrinov,* Anatoli Muravski, ${ }^{\dagger}$ and Hoi Sing Kwok \\ Hong Kong University of Science and Technology, Clear Water Bay, Kowloon, Hong Kong
}

Hirokazu Takada, Hidenari Akiyama, and Haruyoshi Takatsu

Dainippon Ink and Chemicals Incorporated, 631, Sakato, Sakura-shi, Chiba-ken, Japan

(Received 17 June 2003; revised manuscript received 4 September 2003; published 15 December 2003)

\begin{abstract}
The photoinduced alignment of a liquid crystal (LC) on films of azo dyes was studied for the liquid crystal display applications. In order to improve the alignment stability of the photoaligned dye (SD-1), the azo-dye derivative with polymerizable terminal groups was synthesized (SDA-1). The films of SDA-1 exhibit good photoalignment properties and ultraviolet stability after thermal polymerization. We investigated the anchoring coefficient for polar and azimuthal energy for both azo-dyes SD-1 and SDA-1 using a differential method developed by us. The polar and azimuthal anchoring strengths of azo-dye aligning materials are comparable with those of usual polyimide aligning materials. The electro-optic response of a $90^{\circ}$ twisted LC cell remains almost the same after heating up to $200^{\circ} \mathrm{C}$, i.e., the anchoring energy remains considerably high.
\end{abstract}

DOI: 10.1103/PhysRevE.68.061702

PACS number(s): 61.30.Hn

\section{INTRODUCTION}

The photoinduced alignment of a liquid crystal (LC) on azo-dye films have been studied for the liquid crystal display applications [1-3]. The azo-dye molecules are photochemically stable and align their absorption oscillator perpendicular to the polarization of the activating ultraviolet (UV)-light without cis-trans isomerization process [4-6]. This process was described statistically as a diffusion motion of dye molecules under the action of a polarized light $[7-10]$. The order parameter induced in the azo-dye film is considerably high and the LC polar and azimuthal anchoring energy is as large as that one of the rubbed polyimide layer [3]. Furthermore, high-thermal stability of the alignment and high-voltage holding ratio in the LC devices using the film suggest potentialities of its practical use.

In order to improve durability against light exposure and moisture, we developed an azo-dye monomer, which can be polymerized by heating after the photoalignment. We propose a new differential method of measuring polar anchoring energy and discuss the anchoring properties of azo-dye layers. The thermopolymerized azo-dye layers are UVstable and provide a high LC aligning quality.

\section{EXPERIMENT}

The dye compound, synthesized for the studies of photoinduced alignment, is shown in Fig. 1. SD1 was synthesized from corresponding benzidinedisulfonic acid using azo coupling. The product was purified by recrystallization. The azodye SD1 was dissolved in N,N-dimethylformamide (DMF) at a concentration of $1 \mathrm{wt} \%$. The solution was spin coated onto glass substrates with indium-tin-oxide electrodes and dried at $100^{\circ} \mathrm{C}$. The coated film on the substrate was uniform

\footnotetext{
*Email address: eechigr@ust.hk

${ }^{\dagger}$ Permanent address: Institute of the Applied Physique Problems, Kurchatova 7, Minsk, Belarus.
}

and about $10-20 \mathrm{~nm}$ thick. UV light was irradiated onto the surface of the film using super-high-pressure $\mathrm{Hg}$ lamp through an interference filter at $365 \mathrm{~nm}$. The light intensity irradiated on the surface of the film was $5-15 \mathrm{~mW} / \mathrm{cm}^{2}$ for polarized light and $40 \mathrm{~mW} / \mathrm{cm}^{2}$ for nonpolarized light. Two glass substrates with the photoaligned films were assembled to form liquid crystal cells to measure the azimuthal and polar anchoring energy. The cell thickness was 5.7 and 22 $\mu \mathrm{m}$, correspondingly. After heating at $150^{\circ} \mathrm{C}$ for $1 \mathrm{~h}$, liquid crystal mixtures ZLI 5700-000 (from Merck) were injected into the cell in an isotropic phase by capillary action.

The polymerizable azo-dye SDA-1 shown in Fig. 2 was also synthesized. It was dissolved in DMF and a heat initiator V-65 (from Wako pure chemical industries, Ltd.,) was added in relation of 1:50 to SDA-1. The mixture was spin coated onto the glass substrates, dried at $100{ }^{\circ} \mathrm{C}$ and photoaligned in the same manner as SD-1 [1-3]. After the photoaligning procedure, the SDA-1 films were heated at $150^{\circ} \mathrm{C}$ for polymerization.

\section{MEASURING OF POLAR ANCHORING ENERGY BY A DIFFERENTIAL METHOD}

In a differential method we have found the conditions, when we can exclude the contribution of the bulk deformation into the measured value of the LC layer phase retardation. Let us compare the retardations of the two LC cells having the same thickness. If the anchoring energy in the two cells is the same, the phase retardations will be also equal for any voltage applied. If anchoring properties in these cells are different, the difference in the phase retardations will be proportional to the difference in the values of the corresponding

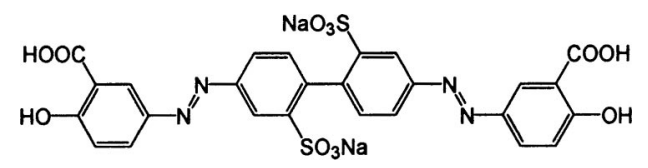

FIG. 1. Sulfuric azo-dye SD1 used for the study of photoalignment. 


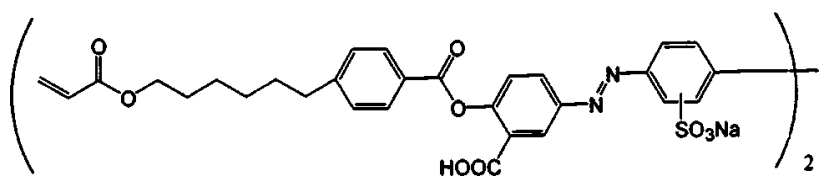

FIG. 2. Polymerizable azo-dye SDA-1.

anchoring energies. The LC phase retardation dependence versus electric field can be defined from the high-electricfield technique [11-13]. The dielectric properties of the alignment layers must be also allowed for, which is shown on the equivalent electrical circuit of the LC cell (Fig. 3). The normalized phase retardation $R / R_{0}$ in this case [11-13]

$$
\frac{R}{R_{0}}=\left(\frac{1}{C}+\frac{2}{C_{\mathrm{al}}}\right) C_{\perp} I_{0} \frac{V_{C}}{V}-2 \frac{K_{11}}{W d}
$$

where

$$
\begin{aligned}
I_{0}= & \frac{2}{\pi} \int_{0}^{\pi / 2} \frac{1-\nu+\sqrt{1-\nu}}{1-\nu \cos ^{2} \varphi+\sqrt{1-\nu \cos ^{2} \varphi}} \\
& \times \sqrt{\left(1+k \sin ^{2} \varphi\right)\left(1-\gamma \cos ^{2} \varphi\right)} \cos \varphi d \varphi, \\
\gamma= & \frac{\varepsilon_{\|}-\varepsilon_{\perp}}{\varepsilon_{\perp}}, \quad k=\frac{K_{33}-K_{11}}{K_{11}}, \quad \nu=\frac{n_{e}^{2}-n_{o}^{2}}{n_{e}^{2}},
\end{aligned}
$$

$W$ is a polar anchoring energy; $d$ is a LC layer thickness; $\varepsilon_{\|}$, $\varepsilon_{\perp}, \Delta \varepsilon=\varepsilon_{\|}-\varepsilon_{\perp}, K_{11}, K_{33}, n_{e}, n_{\mathrm{o}}$ are a LC dielectric, elastic, and optical constants, respectively, $C, C_{\perp}$, and $C_{\text {al }}$ are capacities of the LC layer under the voltage $V$, infinite voltage, and the capacitance of the aligning layer, accordingly, $V_{C}$ is critical (threshold) voltage. In the $R V$ method, the capacity has the following voltage dependence [13]:

$$
C=C_{\|} \frac{1-\alpha \frac{\Delta \varepsilon}{\varepsilon_{\|}} \frac{V_{C}}{V}}{1-\frac{2 K_{11}}{W d} \frac{\Delta \varepsilon}{\varepsilon_{\perp}}},
$$

where $\quad \alpha=1 / \pi \int_{\sin ^{2} \theta_{p}}^{1} \sqrt{[(1+\gamma)(1+k \eta)] /[\eta(1+\gamma \eta)]} d \eta$, $\theta_{\mathrm{p}}$-LC pretilt angle at the substrates. We can rewrite Eqs. (1) and (2) as Eq. (3)

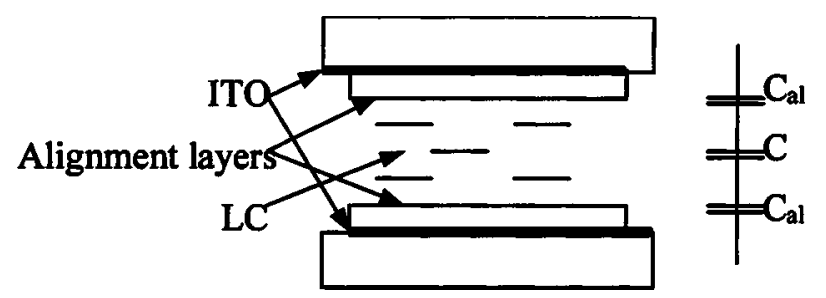

FIG. 3. An equivalent electrical circuit of the LC cell. $C$ and $C_{\mathrm{al}}$ capacities of the LC layer and the aligning layer accordingly (1).

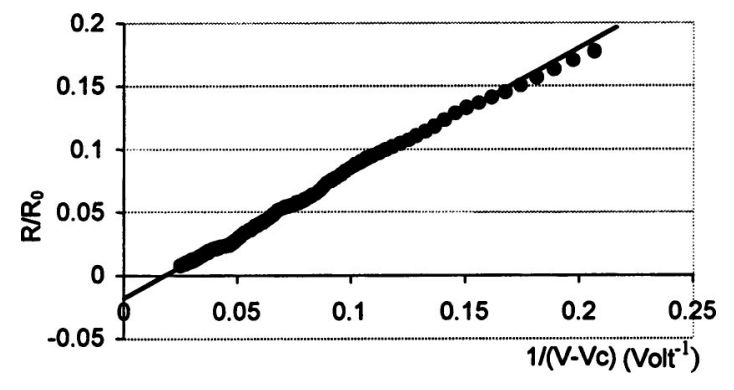

FIG. 4. Retardation $R / R_{0}$ (3) versus inverse voltage $1 /\left(V-V_{C}\right)$, where $V_{C}$ is the critical (threshold) voltage for SD-1 photoalignment materials. The UV exposure time was $30 \mathrm{~min}$; the thickness of the LC cell (ZLI 5700-000) was $5.7 \mu \mathrm{m}$. The measured polar anchoring coefficient $W=W_{\text {polar }}=4.7 \times 10^{-4} \mathrm{~J} / \mathrm{m}^{2}$.

$$
\begin{aligned}
& \frac{R}{R_{0}}=\left(\frac{\frac{\varepsilon_{\perp}}{\varepsilon_{\|}}}{1-\alpha \frac{\Delta \varepsilon}{\varepsilon_{\|}} \frac{V_{0}}{V}}+2 \frac{\varepsilon_{\perp}}{\varepsilon_{\mathrm{al}}} \frac{d_{\mathrm{al}}}{d}\right) I_{0} \frac{V_{C}}{V}-\frac{2 K_{11}}{W d} \\
& \times\left(1+\frac{\frac{\Delta \varepsilon}{\varepsilon_{\|}} I_{0} \frac{V_{C}}{V}}{1-\alpha \frac{\Delta \varepsilon}{\varepsilon_{\|}} \frac{V_{C}}{V}}\right) .
\end{aligned}
$$

The complicated dependence (3) in the limit of high voltages, $V \rightarrow \infty$, becomes

$$
\frac{R_{\infty}}{R_{0}}=-2 \frac{K_{11}}{W d}
$$

If we cannot apply a high voltage, the value of $R_{\infty}$ is not easy to find. In this case we have to modify the above-mentioned differential method to obtain the anchoring energy. If we have two cells with different anchoring properties and different thickness, the relative differential phase retardation writes (indices 1 and 2 refer to the first and the second cell, respectively)

$$
\begin{aligned}
& \frac{R^{1}}{R_{0}^{1}}-\frac{R^{2}}{R_{0}^{2}}=2 \varepsilon_{\perp}\left(\frac{d_{\mathrm{al}}^{1}}{\varepsilon_{\mathrm{al}}^{1} d^{1}}-\frac{d_{\mathrm{al}}^{2}}{\varepsilon_{\mathrm{al}}^{2} d^{2}}\right) I_{0} \frac{V_{C}}{V}-2 K_{11} \\
& \times\left(1+\frac{\frac{\Delta \varepsilon}{\varepsilon_{\|}} I_{0} \frac{V_{C}}{V}}{1-\alpha \frac{\Delta \varepsilon}{\varepsilon_{\|}} \frac{V_{C}}{V}}\right)\left(\frac{1}{W^{1} d^{1}}-\frac{1}{W^{2} d^{2}}\right) .
\end{aligned}
$$

In the right part of Eq. (5) we have the sum of "dielectric" and "anchoring" contributions. If the thickness of the alignment layer is small: $\mathrm{d} / \varepsilon_{\perp} \gg \mathrm{d}_{\mathrm{al}} / \varepsilon_{\text {al }}$, the "dielectric" term disappears.

The expression (5) can be written in more simple form for a high-voltage $V>6 V_{\text {th }}$, taking into account Eqs. (1) and (2) 


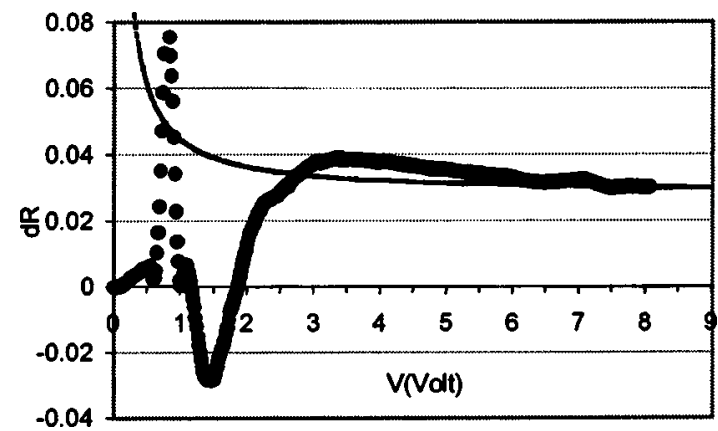

FIG. 5. Difference of the normalized retardations dR (7) in two cells versus voltage $V$ for SDA-1 photoalignment material. The thickness of the first LC cell (ZLI 5700-000) was $22 \mu \mathrm{m}$ and the second $5.7 \mu \mathrm{m}$. The UV light exposure time was $30 \mathrm{~min}$. The measurement was performed before polymerization. The dotted and solid lines show the results of the measurements and approximation by formula (7), respectively. The measured polar anchoring energy $W_{\text {polar }}=2.3 \times 10^{-4} \mathrm{~J} / \mathrm{m}^{2}$.

$$
\frac{R^{1}}{R_{0}^{1}}-\frac{R^{2}}{R_{0}^{2}}=-2 K_{11}\left(1+\frac{\Delta \varepsilon}{\varepsilon_{\|}} I_{0} \frac{V_{C}}{V}\right)\left(\frac{1}{W^{1} d^{1}}-\frac{1}{W^{2} d^{2}}\right) .
$$

Expression (6) is a basic formula for the differential method. If we prepared the two cells with identical alignment layers but different thickness $d^{1}$ and $d^{2}$, the expression (6) will transform to Eq. (7) and helps to find the LC polar anchoring energy $W$

$$
d R=\frac{R^{1}}{R_{0}^{1}}-\frac{R^{2}}{R_{0}^{2}}=-2 \frac{K_{11}}{W}\left(1+\frac{\Delta \varepsilon}{\varepsilon_{\|}} I_{0} \frac{V_{C}}{V}\right)\left(\frac{1}{d^{1}}-\frac{1}{d^{2}}\right) .
$$

\section{RESULTS AND DISCUSSION}

We investigated the anchoring properties of SD-1 and SDA-1 using $R V$ high-voltage technique [13] and our differential method for the measurement of polar anchoring energy of azo-dye aligning layers. The azimuthal anchoring energy was measured by the method, described in Ref. [14].

Figure 4 shows the retardation of LC cell versus applied voltage for SD-1 photoalignment materials. The polar anchoring coefficient for this material was $W=W_{\text {polar }}=4.7$
TABLE I. Polar and azimuthal anchoring energy of azo-dye photoaligning materials.

\begin{tabular}{lcc}
\hline \hline & $\begin{array}{c}\text { Polar anchoring, } \\
W_{\text {polar }}\left(\mathrm{J} / \mathrm{m}^{2}\right)\end{array}$ & $\begin{array}{c}\text { Azimuthal anchoring, } \\
W_{\text {azimuthal }}\left(\mathrm{J} / \mathrm{m}^{2}\right)\end{array}$ \\
\hline SD-1 & $4.7 \times 10^{-4}$ & $\begin{array}{c}7.2 \times 10^{-5} \\
\left(0^{-4}(\text { Ref. }[3])\right. \\
4.2 \times 10^{-6}\end{array}$ \\
$\begin{array}{c}\text { SDA-1 before } \\
\text { polymerization }\end{array}$ & $2.3 \times 10^{-4}$ & $2.2 \times 10^{-5}$ \\
$\begin{array}{c}\text { SDA-1 after } \\
\text { polymerization }\end{array}$ & $8.5 \times 10^{-4}$ & $\begin{array}{c}3.29 \times 10^{-5} \text { (maximum value, } \\
\text { obtained in our experiments) }\end{array}$ \\
\hline
\end{tabular}

$\times 10^{-4} \mathrm{~J} / \mathrm{m}^{2}$. We measured the similar dependence for SDA-1 photoalignment material with $W_{\text {polar }}=8.5$ $\times 10^{-4} \mathrm{~J} / \mathrm{m}^{2}$ (Fig. 5).

We determined the polar anchoring energy before the polymerization of SDA-1 material. The high-voltage technique cannot be used for such measurements, because the alignment layer before polymerization is not stable and the LC pretilt angle increases and does not return to its initial value. In this case we used the modified differential method to find the polar anchoring coefficient (Fig. 5). This method described above has many limitations in comparison with a high-voltage technique, but enables one to measure a polar anchoring energy even for rather small applied voltage (slightly more than $6 V_{C}$ ). The measured values of the azimuthal anchoring energy are also given in Table I.

The polymerization procedure in SDA-1 is very sensitive to the heating rate, when we increase the temperature to the polymerization starting point $T=150{ }^{\circ} \mathrm{C}$. We are facing the two competing processes in this case: (i) disordering process with a large time constant, that decrease with temperature and (ii) polymerization process, which stabilize the layer ordering, but starts only when the system comes to $T$ $=150{ }^{\circ} \mathrm{C}$. If fast heating is provided, the LC anchoring energy assumes its maximum value. Thus, when heating the SDA-1 layer during $5 \mathrm{~min}$, we got the azimuthal anchoring energy $W_{\text {azimuthal }}=2.2 \times 10^{-5} \mathrm{~J} / \mathrm{m}^{2}$ (see also Table I). At the same time while heating the SDA-1 layer for $3 \mathrm{~h}$, we observe the system degradation and even see some Schlieren textures inside the cell. This means that the azimuthal anchoring energy is very small.

TABLE II. Anchoring energy of photoaligned LC cells.

\begin{tabular}{lcc}
\hline \hline \multicolumn{1}{c}{ Photoaligned material } & Polar anchoring $\left(\mathrm{J} / \mathrm{m}^{2}\right)$ & Azimuthal anchoring $\left(\mathrm{J} / \mathrm{m}^{2}\right)$ \\
\hline $\begin{array}{l}\text { Linearly photopolymerized (LPP) } \\
\text { material LPPF 301 CP (Rolic) [15] }\end{array}$ & $4.8-7 \times 10^{-6}$ \\
$\begin{array}{l}\text { Polyvinylcinnamate (PVCi) [16-19] } \\
\text { Poly (cyclobutanetetracarboxylic acid } \\
\text { dianhydrideoxydianiline)imide } \\
\quad \text { CBDA-ODA) [20] }\end{array}$ & $6-14 \times 10^{-6}$ \\
$\begin{array}{l}\text { Photodimerized molecular monolayers } \\
\text { (PDMLs) [21] }\end{array}$ & $0.1-1 \times 10^{-6}$ \\
\hline \hline
\end{tabular}




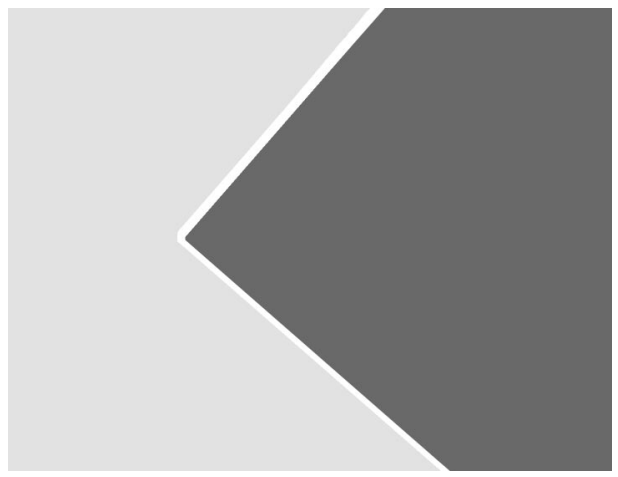

FIG. 6. Polarized microscope photograph of LC alignment on photoaligned polymerized SDA-1 film [23]. The polymerization was performed for $1 \mathrm{~h}$ at $T=150^{\circ} \mathrm{C}$. The size of the picture is 1 $\mathrm{mm}^{2}$. Both the homogeneous and twist LC alignments look perfect between crossed polarizers. The LC cell thickness was $10 \mu \mathrm{m}$.

However, the situation can be considerably improved by the second UV illumination of SDA-1 layers. After 30 min of UV illumination we again obtained the azimuthal energy of SDA-1c layer $W=W_{\text {azimuthal }}=3.1 \times 10^{-5} \mathrm{~J} / \mathrm{m}^{2}$. We did not observe the similar changes in the value of the SDA-1 polar anchoring energy for various rates of the heating up to the point of the polymerization.

The values of polar and azimuthal anchoring energies on photoaligned azo-dye surfaces of SD-1 and SDA-1 (after polymerization) provided in Table I are about an order of magnitude higher, which is usually measured for photoaligned LC cells [15-21] (Table II). The azimuthal anchoring energy is an order of magnitude lower than the polar one, which is usually the case [22]. The mechanism of LC-azo-dye layer interaction is not yet clarified. We believe that dipole-dipole interaction does not play such an important role as, e.g., in case of photodimerized molecular monolayers (PDMLs) [21], so dispersion forces and induced dipole interactions have to be allowed for. The latter is clear from Table I, which states that the polymerization process of SD-1 layer results in a considerable increase both of polar and azimuthal anchoring energy, while dipole-dipole interactions remain almost the same.

SDA-1 film can provide a high LC aligning quality, using either polarized or obliquely incident nonpolarized light irradiation [23] (Fig. 6). LC on the polymerized SDA-1 film was aligned perpendicular to the polarization of the UV light, as it was in the case of photoaligned SD-1 layer [1-3]. The pretilt of LC was achieved by an obliquely incident nonpolarized light illumination [3]. The LC cell alignment was perfect and stable against UV-light exposure and moisture after the polymerization of SDA-1 dye. Figure 7 compares the transmission voltage characteristics of the of $90^{\circ}$ twisted nematic LC cell (TN-LC) based on photoaligned azo-dyes SD-1 and SD-2 films and rubbed polyimide PI 2424 layer [3]. The azodye SD-2 has a similar structure as SD-1 and differs only by the lateral substituents $[3,23]$. The transmission-voltage curves (TVCs) are similar and a very good contrast as the result of a perfect LC alignment can be measured directly. It is greater than 70:1 at the normal incidence. The electro-optical response does not change even in

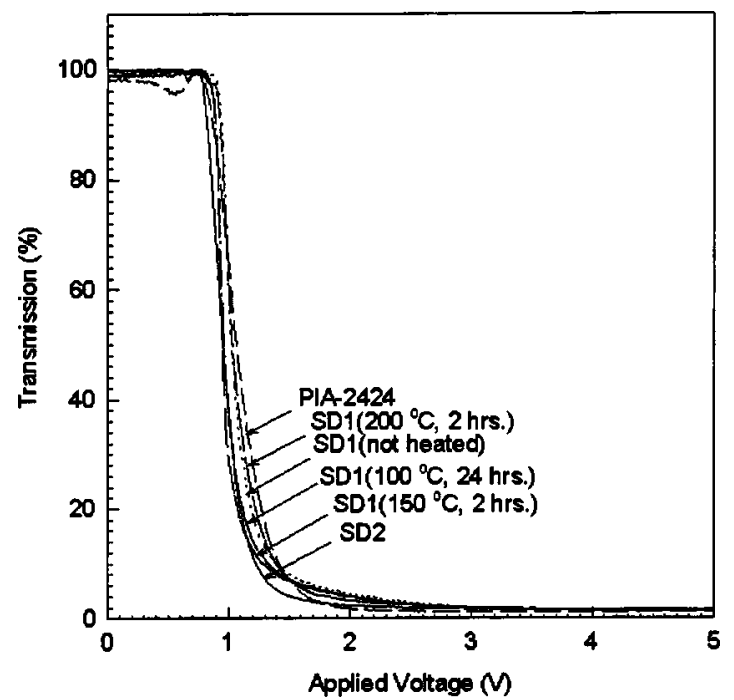

FIG. 7. The comparison of transmission-voltage curves (TVCs) for $90^{\circ}$ twisted nematic LC cell (TN-LC) based on photoaligned azo-dyes SD1 and SD2 films and rubbed polyimide PI 2424 layer [3]. The azo-dye SD-2 has a similar structure as SD-1 and differs only by the lateral substituents $[3,23]$. Heating the cell up to 100 , 150 , and $200{ }^{\circ} \mathrm{C}$ during $2 \mathrm{~h}$ does not have any effect on the transmission-voltage curve. The contrast ratio remains considerably high $(>70: 1)$.

case the SD1 photoaligned cell is heated up to $200{ }^{\circ} \mathrm{C}$ during 2 h, i.e., the anchoring energy remains considerably high. Our preliminary investigations show that the polymerized SDA-1 azo-dye material can provide even higher thermostability. We will publish the detailed results elsewhere.

\section{CONCLUSION}

The photoaligned azo dyes, which exhibit the effect of induced ordering due to the reorientation of the molecules perpendicular to the polarization of the UV light, are promising photoalignment layer for LC devices. SD-1 and SDA-1 azo-dyes exhibit the polar and azimuthal anchoring energies, which is very close to those of the common polyimide (PI) aligning materials. We show also that the fast heating rate of SDA-1 material up to the polymerization point provides the higher values of the LC azimuthal anchoring energy. The values of both LC polar and azimuthal anchoring energy with polymerized SDA-1 layers are about an order of magnitude higher than usually found for photoaligned LC cells and are comparable with rubbed PI layers. The photoaligned SD-1 and SDA-1 layers provide a high thermostability and the corresponding transmission-voltage curve of $90^{\circ}$ twisted nematic LC cell (TN-LC) remains almost the same after heating up to $200{ }^{\circ} \mathrm{C}$, which confirms our results that the anchoring energy is considerably high.

\section{ACKNOWLEDGMENTS}

This research was partially supported by RGC Grant Nos. HKUST6004/01E and HKUST6102/03E. 
[1] W. C. Yip, E. K. Prudnikova, V. M. Kozenkov, V. G. Chigrinov, H. S. Kwok, H. Akiyama, M. Fukuda, H. Takada, and H. Takatsu, SID'01 Digest, p. 1170.

[2] V. Chigrinov, E. Prudnikova, V. Kozenkov, Z. Ling, H. S. Kwok, H. Akiyama, T. Kawara, H. Takada, and H. Takatsu, SID'02 Digest, p. 1106.

[3] V. Chigrinov, E. Prudnikova, V. Kozenkov, H. Kwok, H. Akiyama, T. Kawara, H. Takada, and H. Takatsu, Liq. Cryst. 29, 1321 (2002).

[4] V. M. Kozenkov, S. G. Yudin, E. G. Katyshev, S. P. Palto, V. T. Lazareva, and V. A. Barachevskiy, Pis'ma Zh. Tekh. Fiz. 12, 1267 (1986).

[5] M. I. Barnik, V. M. Kozenkov, N. M. Shtykov, S. P. Palto, and S. G. Yudin, J. Mol. Electron. 5, 53 (1989).

[6] M. Schönhoff, M. Mertesdorf, and M. Löshe, J. Phys. Chem. 100, 7558 (1996).

[7] V. M. Kozenkov and V. A. Barachevskiy, Photosensitive Materials and their Application in Holography (Nauka, Leningrad, 1985), p. 89.

[8] S. P. Palto, N. M. Shtykov, V. A. Khavrichev, and S. G. Yudin, Mol. Mater. 1, 3 (1992).

[9] T. G. Pedersen and P. M. Johansen, Phys. Rev. Lett. 79, 2470 (1997)

[10] V. G. Chigrinov, H. S. Kwok, W. C. Yip, V. M. Kozenkov, E. K. Prudnikova, B. Z. Tang, and F. Salhi, Proc. SPIE 4463, 117 (2001).
[11] H. Yokoyama and H. A. van Sprang, J. Appl. Phys. 57, 4520 (1985).

[12] H. Yokoyama and R. Sun, Jpn. J. Appl. Phys., Part 2 39, L45 (2000).

[13] Yu. A. Nastishin, R. D. Polak, S. V. Shiyanovskii, V. H. Bodnar, and O. D. Lavrentovich, J. Appl. Phys. 86, 4199 (1999).

[14] V. A. Konovalov, A. A. Muravski, and S. Ye. Yakovenko, SID'00 Digest, p. 620.

[15] S.-K. Hahn and D. Kang, IMID’03 Digest, p. 619.

[16] R. Yamaguchi, Yu. Goto, and S. Sato, Jpn. J. Appl. Phys., Part 2 41, L889 (2002).

[17] M. W. Kim, A. Rastegar, I. Drevensek Olenik, and Th. Rasing, J. Appl. Phys. 90, 3332 (2001).

[18] D. Andrienko, F. Barbet, D. Bormann, Yu. Kurioz, S.-B. Kwon, Yu. Reznikov, and M. Warenghem, Liq. Cryst. 27, 365 (2000).

[19] N. Klopsar, I. Drevensek Olenik, M. Copic, M. W. Kim, A. Rastegar, and Th. Rasing, Mol. Cryst. Liq. Cryst. Suppl. Ser. 368, 395 (2001).

[20] M. Hasegawa, Jpn. J. Appl. Phys., Part 2 41, L1167 (2002).

[21] D. Shenoy, L. Beresnev, D. Holt, and R. Shashidhar, Appl. Phys. Lett. 80, 1538 (2002).

[22] L. M. Blinov and V. G. Chigrinov, Electrooptic Effects in Liquid Crystal Materials (Springer-Verlag, New York, 1994).

[23] H. Takada, H. Akiyama, H. Takatsu, V. Chigrinov, E. Prudnikova, V. Kozenkov, and H. S. Kwok, SID’03 Digest, p. 620. 